\title{
Anesthesia Procedure
}

National Cancer Institute

\section{Source}

National Cancer Institute. Anesthesia Procedure. NCI Thesaurus. Code C15181.

Treatment with a pharmacological substance that produces a loss of feeling. 